\title{
The mitochondrial genome of Iberobaenia (Coleoptera: Iberobaeniidae): First rearrangement of protein coding genes in the beetles
}

\author{
Carmelo Andújar, ${ }^{1,2 *}$ Paula Arribas, ${ }^{1,2}$ Benjamin Linard, ${ }^{1}$ Robin Kundrata, ${ }^{3}$ Ladislav Bocak, ${ }^{3}$ \\ Alfried P. Vogler ${ }^{1,2}$ \\ ${ }^{1}$ Department of Life Sciences, Natural History Museum, Cromwell Road, London, SW7 5BD, UK \\ ${ }^{2}$ Department of Life Sciences, Imperial College London, Silwood Park Campus, Ascot, SL5 7PY, UK \\ ${ }^{3}$ Department of Zoology, Faculty of Science, Palacky University, 17. listopadu 50, 77146 Olomouc, Czech \\ Republic
}

Key words: Coleoptera, gene rearrangement, mitochondrial metagenomics, mitochondrion, Elateroidea.

\begin{abstract}
:
The complete mitochondrial genome of the recently discovered beetle family Iberobaeniidae is described and compared with known coleopteran mitogenomes. The mitochondrial sequence was obtained by shotgun metagenomic sequencing using the Illumina Miseq technology and resulted in an average coverage of 130x and a minimum coverage of 35x. The mitochondrial genome of Iberobaeniidae includes 13 protein-coding genes, 2 rRNAs, 22 tRNAs genes and one putative control region, and showed a unique rearrangement of protein coding genes. This is the first rearrangement affecting the relative position of protein-coding and ribosomal genes reported for the order Coleoptera.
\end{abstract}

Running Title: The mitochondrial genome of Iberobaenia 
Coleoptera are the most diverse order of insects, with about 179 extant families (Bouchard et al. 2011) and more than 350000 described species. More than 250 complete or almost complete mitochondrial genomes have been sequenced to date, including representatives from $\approx 100$ families. Despite the high diversity and ancient origin of Coleoptera, estimated at around 300 MYA (Hunt et al., 2007; Misof et al., 2014), all known coleopteran mitogenomes share the same gene order and orientation for the protein-coding and rRNA genes, while rearrangements found to date affect only the position of the tRNA genes (Timmermans \& Vogler, 2012). The genus Iberobaenia Bocak, Kundrata, Andújar \& Vogler 2015 has been recently discovered and phylogenetic analyses conducted on mitochondrial and nuclear genes revealed the distinctiveness of the new lineage within the superfamily Elateroidea, resulting in the description of a new beetle family, Iberobaeniidae (Bocak et al.in press).

For sequencing the complete mitochondrial genome of Iberobaenia we followed the mitochondrial metagenomic approach (MMG) (Dettai et al., 2012; Zhou et al., 2013; Andújar et al., 2015; Crampton-Platt et al., 2015), which allows to assemble complete mitochondrial genomes from shotgun sequencing of specimen mixtures. The raw DNA extracted from a larval specimen of Iberobaenia sp. (Sierra de Cabra, Córdoba, Spain; Voucher specimen deposited in the Natural History Museum of London-BMNH 1042541) was pooled in equimolar concentration with DNA from 20 other Coleoptera from distant lineages mostly in the suborder Adephaga not connected to the current study. The dsDNA concentration was measured using a Qubit 2.0 Fluorometer (Life Technologies Corp., Carlsbad, CA, USA). A TruSeq DNA library was constructed with the pooled DNA and sequenced using the Illumin aMiSeq platform (2x300 bp; 800-950 bp insert size) in 45\% of an Illumina flow cell.

The Illumina output was quality assessed with FastQC 0.10.1 (Babraham Institute 2013) and processed with Trimmomatic 0.30 (Lohse et al. 2012) for Illumina adapter removal. Reads we subsequently filtered using Blast 2.2.27 (Altschul et al. 1990) against a reference database including 245 nearly complete coleopteran mitochondrial genomes retrieved from GenBank. Surviving mitochondrial reads were subsequently assembled using Celera Assembler 7.0 (Myers 2000), Newbler 2.7 (Margulies et al. 2006) and IDBA-UD (Peng et al. 2012). Celera analyses were done using the RunCa command with the bogart method for unitigger computations, and Unitig Repeat/Unique Toggling was conducted from unitigs longer than 500 bp; other parameters were set to default options. Newbler analyses were run using -mi 95 -ml 150 and IDBA using --maxk 300 --mink 50. 
Mitochondrial contigs longer than 500 bp obtained with the three assemblers were pooled and super-contiged using Geneious 7.1.9 (http://www.geneious.com), saving the 50\% majority rule consensus from the resulting contigs. The mitogenome of Iberobaenia was identified using the cox1 barcode sequence obtained from the same specimen with PCRSanger sequencing (Genbank accession no. XXX), and was annotated using gene predictions with MITOS (Bernt et al., 2013) and manually refined in Geneious. Additionally, mitochondrial Illumina reads were mapped against the mitogenome of Iberobaenia in Geneious (using a 99\% similarity threshold).

The widely overlapping contigs obtained from the three independent assemblers, after super-contiging and circularization, resulted in a complete mitochondrial sequence of 16931bp (Accession no. KT825140) including 13 protein-coding, 2 rRNAs and 22tRNAs genes. The overall base composition was $46.9 \%$ for $\mathrm{A}, 14.1 \%$ for $\mathrm{C}, 7.5 \%$ for $\mathrm{G}, 31.5 \%$ for $\mathrm{T}$. All protein-coding genes were encoded on the H-strand with exception of nad1, nad4, nad4l and nad5. All tRNA genes were encoded on the H-strand with the exception of tRNA-Cys, tRNA-Phe, tRNA-His, tRNA-Val, tRNA-Gln, tRNAPro and tRNA-Leu2. Our predictive annotation resulted in the canonical ATN start codon (isoleucine) for all genes (ATA: nad2, cox1, cob, atp6, atp8 and nad3; ATG: cox2 and cox3; ATT: nad5, nad4 and nad4l; ATC: nad6) but nad1, where a TTG start codon was identified as previously reported in other Coleoptera (Sheffield et al., 2008). With regard to the 3' end, nad2 was predicted with an incomplete stop codon "T", three genes showed the stop codon TAG (nad5, nad1 and cob) and all other genes were inferred with the stop codon TAA. The longest gene was nad5 (1647bp) and the shortest atp8 (150bp). A maximum-likelihood phylogenetic tree was obtained in RaxML (Stamatakis, 2006) including some of the closest relatives to Iberobaniidae according to Bocak et al. (in review) (Fig. 1). The best tree was selected over 100 alternative runs and node support was calculated with 10000 bootstrap replicates.

The structure of the mitogenome of Iberobaenia showed a unique gene rearrangement with regard to the constant pattern found in all other mitochondrial genomes for the order Coleoptera (Timmermans \& Vogler, 2012). The rearrangement affected the protein-coding, ribosomal and tRNAs genes. The gene order for the mitogenome of Iberobaenia sp., including tRNAs, is: I, M, nad2, W,C , Y, cox1, L1, cox2, K, D, atp8, atp6, cox3, nad3, N, R, A, E, F, G, S, nad5, H, nad4l, nad1, control region, Q, nad4, T, P, nad6, cob, S2, L2, rrnL, V, $r r n S$. The high sequencing read coverage (average:130x; max: 213x; min: 35x) provides high reliability to the obtained sequence. This is the first mitochondrial gene rearrangement reported in the order Coleoptera excluding those affecting only to tRNAs (Timmermans \& 
Vogler, 2012). This finding adds to the unique features of the enigmatic beetle family Iberobaeniidae recently described from the Iberian Peninsula.

\section{Acknowledgements}

We thank Alex Aitken, Kevin Hopkins and Peter Foster (Natural History Museum) for their technical assistance. This work was funded by the Leverhulme Trust (grant F/00696/P), the NHM Biodiversity Initiative and the Natural Environment Research Council (grant NE/M021955). PA was supported by a postdoctoral grant from The Royal Society (Newton International Program, UK).

\section{Declaration of interest}

The authors report no conflict of interest. The authors alone areresponsible for the content and writing of the paper.

\section{References:}

Altschul SF, Gish W, Miller W, Myers E.W, Lipman DJ, (1990). Basic local alignment search tool. J Mol Biol 215: 403-10.

Andújar C, Arribas P, Ruzicka F, Crampton-Platt A, Timmermans MJTN, Vogler AP. (2015). Phylogenetic community ecology of soil biodiversity using mitochondrial metagenomics. Mol Ecol 24: 3603-3617.

Babraham Institute (2013). FastQC: A quality control tool for high throughput sequence data. http://www.bioinformatics.babraham.ac.uk/projects/fastqc.

Bernt M, Donath A, Jühling F, Externbrink F, Florentz C, Fritzsch G, Pütz J, Middendorf M, Stadler PF. (2013). MITOS: Improved de novo metazoan mitochondrial genome annotation. Mol Phylogenet Evol 69: 313-319.

Bocak L, Kundrata R, Andújar C, Vogler AP. (2015). The discovery of Iberobaeniidae (Coleoptera: Elateroidea), a new family of beetles from Spain, with immatures detected by environmental DNA sequencing. Proc. R. Soc. London B. Submitted.

Bouchard P, Bousquet Y, Davies A.E, Alonso-Zarazaga M. a, Lawrence JF, Lyal CHC, Newton AF, Reid CAM, Schmitt M, Slipiński SA, Smith ABT. (2011). Family-group names in Coleoptera (Insecta). ZooKeys 972: 1-972.

Crampton-Platt A, Timmermans MJTN, Gimmel ML, Kutty SN, Cockerill TD, Vun Khen C, Vogler AP. (2015). Soup to tree: the phylogeny of beetles inferred by mitochondrial metagenomics of a Bornean rainforest sample. Mol Biol Evol 32: 2302-2316.

Dettai A, Gallut C, Brouillet S, Pothier J, Lecointre G, Debruyne R. (2012). Conveniently pre-tagged and pre-packaged: extended molecular identification and metagenomics using complete metazoan mitochondrial genomes. PloS one 7: e51263. 
Hunt T, Bergsten J, Levkanicova Z, Papadopoulou A, John OS, Wild R, Hammond PM, Ahrens D, Balke M, Caterino MS, Gómez-Zurita J, Ribera I, Barraclough T.G, Bocakova M, Bocak L, Vogler AP. (2007). A comprehensive phylogeny of beetles reveals the evolutionary origins of a superradiation. Science 318: 1913-6.

Lohse M, Bolger AM, Nagel A, Fernie AR, Lunn JE, Stitt M, Usadel B. (2012). RobiNA: a userfriendly, integrated software solution for RNA-Seq-based transcriptomics. Nucleic Acids Res 40: W622-7.

Margulies M, Egholm M, Altman W.E, Attiya S, Bader JS, Bemben LA, Berka J, Braverman MS, Chen Y, Chen Z, Dewell B, Du L, Fierro JM, Gomes XV, Goodwin BC, He W, Helgesen S, Ho C.H, Irzyk GP, Jando SC, Maria L.I, Jarvie TP, Jirage KB, Kim J, Knight JR, Lanza R, Leamon JH, Lefkowitz SM, Lei M, Li J, Kenton L, Lu H, Makhijani VB, Mcdade KE, Mckenna MP, Myers W, Nickerson E, Nobile JR, Plant R, Puc BP, Ronan T, Roth GT, Sarkis GJ, Simons JF, Simpson JW, Srinivasan M, Tartaro KR, Tomasz A, Vogt KA, Greg A, Wang SH, Wang Y, Weiner MP, Yu P, Richard F, Rothberg JM. (2006). Genome sequencing in open microfabricated high density picoliter reactors. Nature 437: 376-380.

Misof B, Liu S, Meusemann K, Peters RS, Donath a, Mayer C, Frandsen PB, Ware J, Flouri T, Beutel RG, Niehuis O, Petersen M, Izquierdo-Carrasco F, Wappler T, Rust J, Aberer a. J, Aspock U, Aspock H, Bartel D, Blanke a, Berger S, Bohm a, Buckley TR, Calcott B, Chen J, Friedrich F, Fukui M, Fujita M, Greve C, Grobe P, Gu S, Huang Y, Jermiin LS, Kawahara AY, Krogmann L, Kubiak M, Lanfear R, Letsch H, Li Y, Li Z, Li J, Lu H, Machida R, Mashimo Y, Kapli P, McKenna DD, Meng G, Nakagaki Y, Navarrete-Heredia JL, Ott M, Ou Y, Pass G, Podsiadlowski L, Pohl H, von Reumont BM, Schutte K, Sekiya K, Shimizu S, Slipinski A, Stamatakis A, Song W, Su X, Szucsich NU, Tan M, Tan X, Tang M, Tang J, Timelthaler G, Tomizuka S, Trautwein M, Tong X, Uchifune T, Walzl MG, Wiegmann BM, Wilbrandt J, Wipfler B, Wong TKF, Wu Q, Wu G, Xie Y, Yang S, Yang Q, Yeates DK, Yoshizawa K, Zhang Q, Zhang R, Zhang W, Zhang Y, Zhao J, Zhou C, Zhou L, Ziesmann T, Zou S, Xu X, Yang H, Wang J, Kjer KM, Zhou X. (2014). Phylogenomics resolves the timing and pattern of insect evolution. Science 346: 763-767.

Myers EW. (2000). A whole-genome assembly of Drosophila. Science 287: 2196-2204.

Peng Y, Leung HCM, Yiu SM, Chin FYL. (2012). IDBA-UD: a de novo assembler for single-cell and metagenomic sequencing data with highly uneven depth. Bioinformatics 28: 1420-8.

Sheffield NC, Song H, Cameron SL, Whiting MF. (2008). A comparative analysis of mitochondrial genomes in Coleoptera (Arthropoda: Insecta) and genome descriptions of six new beetles. Mol Biol Evol 25: 2499-509.

Stamatakis A. (2006) RAxML-VI-HPC: maximum likelihood-based phylogenetic analyses with thousands of taxa and mixed models. Bioinformatics 22: 2688-90. 
Timmermans MJTN, Vogler AP. (2012). Phylogenetically informative rearrangements in mitochondrial genomes of Coleoptera, and monophyly of aquatic elateriform beetles (Dryopoidea). Mol Phylogenet Evol, 63, 299-304.

Zhou X, Li Y, Liu S, Yang Q, Su X, Zhou L, Tang M, Fu R, Li J, Huang Q. (2013). Ultra-deep sequencing enables high-fidelity recovery of biodiversity for bulk arthropod samples without PCR amplification. GigaScience 2: 1-12.

\section{Figure captions:}

Figure 1. Maximum-likelihood phylogenetic tree including some of the closest relatives to Iberobaniidae according to Bocak et al. (in review). Numbers on nodes represent bootstrap support. Right: Schematic representation of Iberobaenia sp. male specimen.

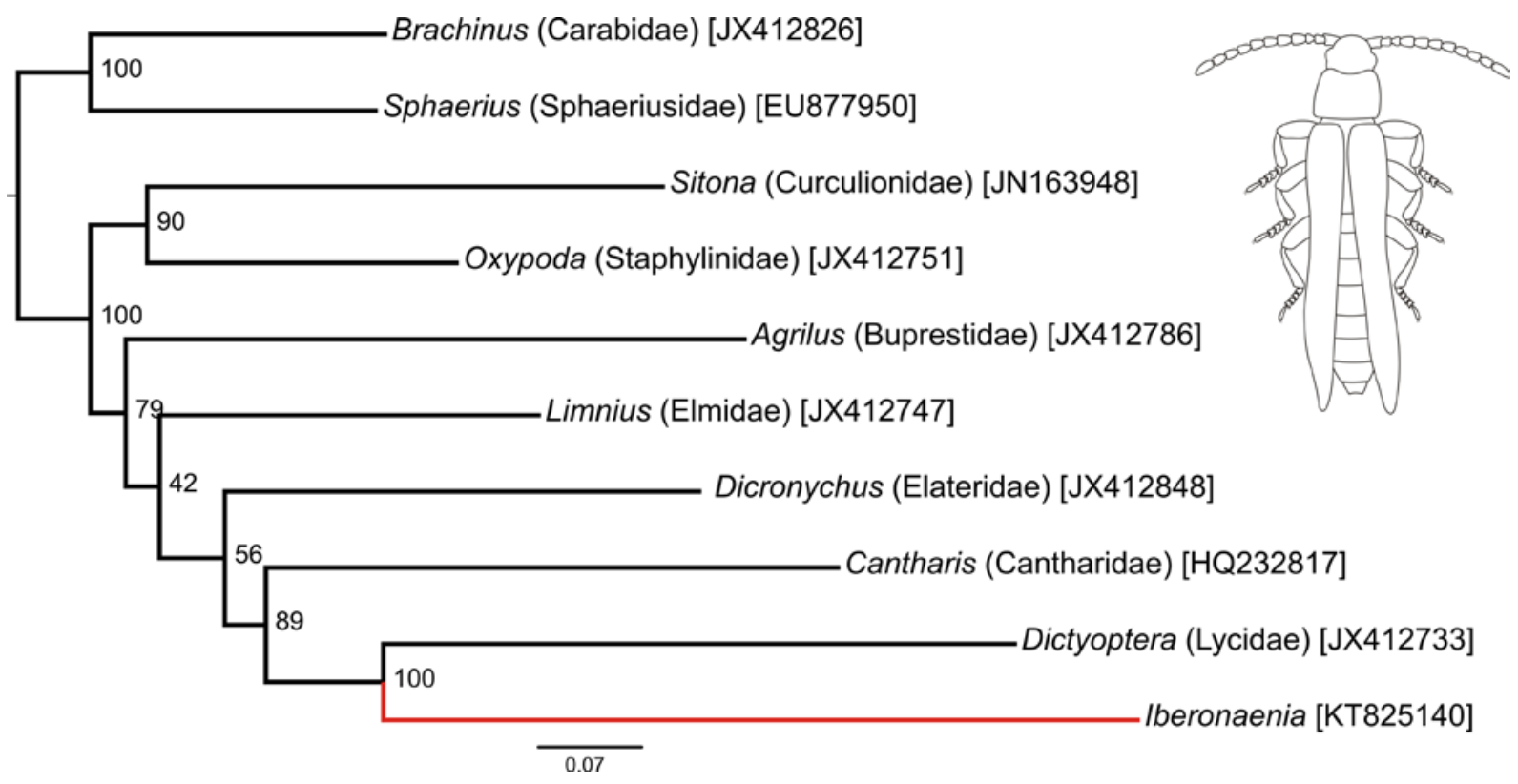

